PROCEEDINGS OF THE

AMERICAN MATHEMATICAL SOCIETY

Volume 138, Number 11, November 2010, Pages 4009-4023

S 0002-9939(2010)10392-1

Article electronically published on June 16, 2010

\title{
ONE-DIMENSIONAL CONTRACTING SINGULAR HORSESHOE
}

\author{
D. CARRASCO-OLIVERA, C. A. MORALES, AND B. SAN MARTÍN
}

(Communicated by Bryna Kra)

\begin{abstract}
In this paper we prove some kind of structural stability defined as usual but restricted to a certain subset of one-dimensional maps coming from first return maps associated to singular cycles for vector fields in manifolds with boundary. The motivation is the stability of the Singular Horseshoes introduced by Labarca and Pacifico where an expanding condition on the singularity holds. Here we obtain analogous result but under a contracting condition.
\end{abstract}

\section{INTRODUCTION}

This paper is motivated by [14, where $C^{1}$ structural stability for vector fields on manifolds with boundary, called Singular Horseshoe, was proved. To do this is necessary to prove $C^{1}$ structural stability for the expanding one-dimensional maps modeling the dynamic. Under a contracting condition on the singularity involved in the Singular Horseshoe [25], this is not at all obvious because the one-dimensional map presents a critical point. Let us state our results in a precise way.

Hereafter we fix two real numbers $a, b$ such that $0<a<b<1$.

Definition 1.1. Define $\mathscr{A}$ as the set of $C^{1}$-maps $f: \operatorname{dom}(f) \subset \mathbb{R} \rightarrow \mathbb{R}$ satisfying the following properties:

(i) $\operatorname{dom}(f)=[0, a] \cup[b, 1]$. Moreover, $f$ is increasing on $[0, a]$ and decreasing on $[b, 1], f(0)=0$ and $|D f(0)|>1, f(1)=0$ and $D f(x)=0$ if and only if $x=1$. Additionally $f(a)>1$ and $f(b)>1$.

(ii) There is a constant $\alpha_{f}>1$ and a continuous positive map $H_{f}:[b, 1] \rightarrow \mathbb{R}$ with $\lim _{x \rightarrow 1}(x-1) D H_{f}(x)=0$ such that

$$
f(x)=|x-1|^{\alpha_{f}} H_{f}(x), \forall x \in[b, 1] .
$$

Note that any map in $\mathcal{A}$ can be perturbed in such a way that the new map presents a hyperbolic sink. Indeed, consider $f \in \mathcal{A}$ and $\delta$ a small positive real

Received by the editors June 11, 2009 and, in revised form, November 26, 2009, January 13, 2010 and January 18, 2010.

2010 Mathematics Subject Classification. Primary 37E05, 37D25; Secondary 37D30, 37F15.

Key words and phrases. One-dimensional maps, conjugation, symbolic dynamic, nonwandering set, transitive set.

The first author was supported in part by Project Mecesup 0202-UCN; Project Fondecyt No. 1040682; Project ADI 17 Anillo en Sistemas Dinámicos de Baja Dimensión, Chile; CONICYT Proyecto Inserción de Nuevos Invertigadores en la Academia, 2009, Folio 79090039.

The second author was partially supported by CNPq, FAPERJ and PRONEX-Brazil.

The third author was partially supported by Project Fondecyt No. 1040682 and Project ADI 17, Anillo en Sistemas Dinámicos de Baja Dimensión, CONICYT - Chile and PRONEX-Brazil. 


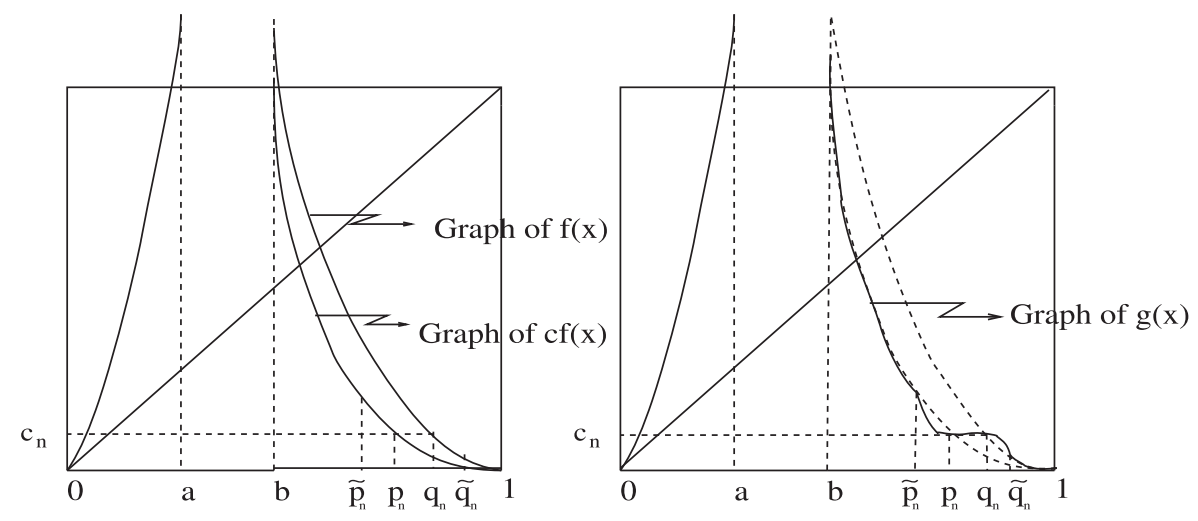

Figure 1. A $C^{1}$-perturbation of $f \in \mathscr{A}$ with a super attractor.

number. For each $n$ consider $c_{n}$ close to 0 and $q_{n}$ close to 1 such that for all $i, 0<i<n, f^{i}\left(c_{n}\right)<a, f^{n}\left(c_{n}\right)=q_{n}$ and $f\left(q_{n}\right)=c_{n}$. Given $c$ smaller than 1 , take $p_{n}$ close to 1 such that $c f\left(p_{n}\right)=f\left(q_{n}\right)=c_{n}$. Note that $p_{n}<q_{n}$. Moreover, if $c$ is close enough to 1 , we can take $\tilde{p_{n}}$ and $\tilde{q_{n}}$ with $\tilde{p_{n}}<p_{n}<q_{n}<\tilde{q_{n}}<1$ such that $2\left|p_{n}-\tilde{p_{n}}\right|=\left|q_{n}-p_{n}\right|=2\left|\tilde{p_{n}}-q_{n}\right|$. Furthermore, for $n$ large enough, we have that $f(x)<\delta$ and $|D f(x)|<\delta$ for all $x \geq \tilde{p_{n}}$. Take a positive bump function $\rho$ defined on $\mathbb{R}$ such that $\rho(x)=0$ for $x$ in the complement of $[-2,2]$ and $\rho(x)=1$ for $x \in[-1,1]$. Then, for $d=\frac{q_{n}+p_{n}}{2}$ we can consider $\psi: \mathbb{R} \rightarrow[0,1]$ given by $\psi(x)=\rho\left(2 \frac{x-d}{q_{n}-p_{n}}\right)$. Then, the map $g$ (see Figure 1) given by

$$
g(x)= \begin{cases}c f(x)(1-\psi(x))+c_{n} \psi(x) & x \leq d \\ f(x)(1-\psi(x))+c_{n} \psi(x) & x \geq d\end{cases}
$$

is $C^{1}$-close to $f$ (namely $|g(x)-f(x)|<2 \delta$ and $|D g(x)-D f(x)|<\delta(1+2 K)$, where $K$ only depends on the map $\rho$ ) and increasing on $[0, a]$ (because $g \equiv f$ at $[0, a])$ and $D g(x) \leq 0$ on $[b, 1]$ by construction. Moreover, $q_{n}$ is a super attracting periodic point for $g$ of period $n+1$.

Now, adding $\epsilon(1-x)^{\alpha_{f}}$ to $g$ for $x \in[b, 1]$ we obtain a new map $\tilde{f} \in \mathcal{A}, C^{1}$-close to $f$ exhibiting a hyperbolic sink. The same construction can be used to obtain maps with many finite or even infinitely many hyperbolic sinks. So, this shows that such maps are not $C^{1}$-stable. However, there is another metric for which these kinds of perturbations are not possible.

As mentioned above, the conditions defining $\mathscr{A}$ are imposed so that they are satisfied by the first return maps associated to Singular Cycles. The following metric in $\mathscr{A}$ retrieves the properties inherited from the proximity of the respective vector fields:

$$
\begin{aligned}
d_{\mathscr{A}}(f, g)= & \max _{x}\left\{\sup _{x}|f(x)-g(x)|, \sup _{x}|D f(x)-D g(x)|,\right. \\
& \sup _{x}\left|H_{f}(x)-H_{g}(x)\right|, \sup _{x}\left|(x-1) \cdot\left(D H_{f}(x)-D H_{g}(x)\right)\right|, \\
& \left.\left|\alpha_{f}-\alpha_{g}\right|: x \in[0, a] \cup[b, 1]\right\} .
\end{aligned}
$$


Definition 1.2. We say that $f, g \in \mathscr{A}$ are topologically conjugated if there is a homeomorphism $h:[0,1] \rightarrow[0,1]$ such that $g \circ h=h \circ f$. We say that $f$ is $\mathscr{A}$ structurally stable if there is a neighborhood $\mathscr{N}$ of $f$ in $\left(\mathscr{A}, d_{\mathscr{A}}\right)$ such that each $g \in \mathscr{N}$ is topologically conjugate to $f$.

Main Theorem. Consider $f \in \mathscr{A}$ such that $(\sqrt{|D f|})^{-1}$ is a convex map on the intervals where it is defined. Then, $f$ is $\mathscr{A}$-structurally stable.

As already mentioned, this result is inspired by [14]. The proof there used the injectivity of the Milnor-Thurston coordinates [21] to construct the conjugacy. In our case, to prove such an injectivity we will need to show the nonexistence of sinks or wandering intervals for small perturbations in $\mathscr{A}$. This is not at all obvious because of the presence of a critical point and our restricted hypothesis about differentiability, and considering the previous examples. To the best of our knowledge, this is not a consequence of any result in the literature. Efforts have been made by a number of authors (see [7, [26, [22, [8], 10, [30, 15], 17, [13, 1], 2], [18, [23], [3], 12], [16], [19] and [29]) towards proving the nonexistence of wandering intervals because their appearance complicates the understanding of the dynamics. They involved some smoothness and other ingredients on the considered maps. In the present case we only require $C^{1}$-smoothness perturbations, so wandering intervals may appear (see [22, [10], 15, [3], [12, 19]: Theorem 2.3, p. 43, 6], 11], [27] and [5]). Afterward, the structural stability will follow from the arguments in [14 (see section 4, pp. 344-345), inspired by Guckenheimer and Williams [9] (see section 2) and Parry 24] (see p. 377).

Finally we would like to acknowledge the referee for pointing out that the theorem is valid under the more general hypothesis about the convexity of $|D f|^{-\frac{1}{2}}$ instead of the negative Schwarzian derivative condition used in a previous version.

\section{Proofs}

To achieve our goal we need to recall the Minimum Principle for maps with negative Schwarzian derivative.

Given a $C^{3}$ map $f \in \mathscr{A}$, the Schwarzian derivative of $f$ at $x \in \operatorname{dom}(f) \backslash\{1\}$ is defined by

$$
S f(x)=\frac{D^{3} f(x)}{D f(x)}-\frac{3}{2}\left(\frac{D^{2} f(x)}{D f(x)}\right)^{2} .
$$

We will say that $f$ has negative Schwarzian derivative whenever $S f(x)<0$ for all $x \neq 1$. It is very well known that if a map has negative Schwarzian derivative, so do all its iterates. Moreover, as $D^{2}\left(|D f(x)|^{-\frac{1}{2}}\right)=-\frac{1}{2}|D f|^{-\frac{1}{2}} S f(x)$, then the strict convexity of $(\sqrt{|D f|})^{-1}$ could be seen as a $C^{1}$ version of the negative Schwarzian condition.

Lemma 2.1 (Minimum Principle). Let $f$ be a $C^{3}$ map with negative Schwarzian derivative and $T=\left[a_{0}, b_{0}\right]$ be a closed interval contained in $\operatorname{dom}(f)$. If $D f(x) \neq 0$ for all $x \in T$, then

$$
|D f(x)|>\min \left\{\left|D f\left(a_{0}\right)\right|,\left|D f\left(b_{0}\right)\right|\right\}, \quad \forall x \in\left(a_{0}, b_{0}\right) .
$$

Of course, for maps with negative Schwarzian derivative the derivative of all its iterates $D f^{i}$ satisfies the Minimum Principle. 
On the other hand, the convexity of $(\sqrt{|D f|})^{-1}$ on $\left[a_{0}, b_{0}\right]$ implies that for all $x \in\left[a_{0}, b_{0}\right]$,

$$
|D f(x)|>\min \left\{\left|D f\left(a_{0}\right)\right|,\left|D f\left(b_{0}\right)\right|\right\} .
$$

Using Lemma 2.1, we can prove that the iterates of $f$ have the same property. Indeed, Cedervall in his $\mathrm{PhD}$ thesis [4] gives a more complete and informative exposition of this topic; in particular, see Lemma 3.1 and Proposition 3.1 in Chapter 3.

Lemma 2.2 (Weak Minimum Principle). Let $f \in \mathscr{A}$ such that $(\sqrt{|D f|})^{-1}$ is a convex map on the intervals where it is defined and $T=\left[a_{0}, b_{0}\right]$ is a closed interval contained in $\operatorname{dom}\left(f^{i}\right)$. If $D f^{i}(x) \neq 0$ for all $x \in T$, then

$$
\left|D f^{i}(x)\right| \geq \min \left\{\left|D f^{i}\left(a_{0}\right)\right|,\left|D f^{i}\left(b_{0}\right)\right|\right\}, \quad \forall x \in\left(a_{0}, b_{0}\right) .
$$

Proof. Take $f$ as in the lemma and fix $i$. We can approximate $f$ by a $C^{3}$ map $g$ such that $(\sqrt{|D g|})^{-1}$ is strictly convex. So $g$ has a negative Schwarzian derivative and $g^{i}$ satisfies the Minimum Principle. Moreover $g$ can be chosen such that $g^{i}$ is very close to $f^{i}$. Hence, if we assume that $f^{i}$ does not satisfy the Minimum Principle, this leads to a contradiction.

The Minimum Principle will be used to find a lower bound, not depending on $i$, for the derivative $\left|D f^{i}(x)\right|$ for all $x$ such that $f^{i}(x)$ is far from 1 and 0 . A similar constant was obtained in 20] for the considered maps (see Lemma 3, equation (8), p. 880).

Lemma 2.3. Let us consider a map $f \in \mathscr{A}$ such that $(\sqrt{|D f|})^{-1}$ is convex and let us consider two real numbers $c$ and $d, 0<c<d<1$. Then there exists a constant $C_{0}=C_{0}(f, c, d)>0$ such that $\forall i \in \mathbb{N}$ and $x \in \operatorname{dom}\left(f^{i}\right)$ with $f^{i}(x) \in[c, d]$ we have that

$$
\left|D f^{i}(x)\right| \geq C_{0}
$$

Proof. Fix $f \in \mathscr{A}$ as in the lemma derivative and two numbers $c$ and $d$ such that $0<c<d<1$.

Define

$$
C_{0}=\min \{c, 1-d\} .
$$

Now, given $i \in \mathbb{N}$ and $x$ such that $f^{i}(x) \in[c, d]$, let us consider $I_{x}=\left[\xi_{0}, \xi_{1}\right]$, the maximal interval containing $x$ where $f^{i}$ is defined. For maximality of $I_{x}$ we have that either $[0, c] \subset f^{i}\left(\left[\xi_{0}, x\right]\right)$ and $[d, 1] \subset f^{i}\left(\left[x, \xi_{1}\right]\right)$, or $[d, 1] \subset f^{i}\left(\left[\xi_{0}, x\right]\right)$ and $[0, c] \subset f^{i}\left(\left[x, \xi_{1}\right]\right)$.

In both cases, by the Mean Value Theorem there are $\tilde{\xi}_{0}, \tilde{\xi}_{1}$ with $\xi_{0}<\tilde{\xi}_{0}<x<$ $\tilde{\xi}_{1}<\xi_{1}$ such that

$$
\left|D f^{i}\left(\tilde{\xi_{0}}\right)\right|=\frac{l\left(f^{i}\left(\left[\xi_{0}, x\right]\right)\right)}{l\left(\left[\xi_{0}, x\right]\right)} \geq l\left(f^{i}\left(\left[\xi_{0}, x\right]\right)\right) \geq C_{0}
$$

and

$$
\left|D f^{i}\left(\tilde{\xi}_{1}\right)\right|=\frac{l\left(f^{i}\left(\left[x, \xi_{1}\right]\right)\right)}{l\left(\left[x, \xi_{1}\right]\right)} \geq l\left(f^{i}\left(\left[x, \xi_{1}\right]\right)\right) \geq C_{0},
$$

where $l(J)$ denotes the length for the interval $J$. 
Using the Minimum Principle (Lemma 2.2), (2.2) and (2.3) we obtain that

$$
\left|D f^{i}(x)\right| \geq \min \left\{\left|D f^{i}\left(\tilde{\xi}_{0}\right)\right|,\left|D f^{i}\left(\tilde{\xi}_{1}\right)\right|\right\} \geq C_{0} ;
$$

therefore the lemma follows.

Figure 2 illustrates the situation for $f^{i}$ for $i=2$.

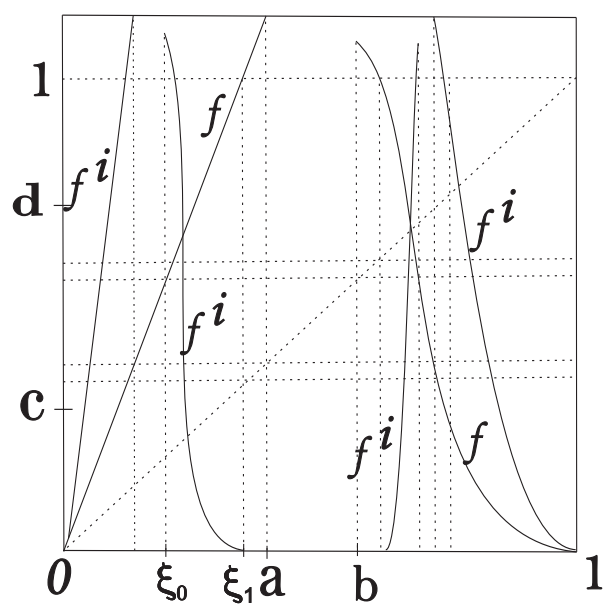

Figure 2. Case $f^{i}$ for $i=2$.

Now we will use Lemma 2.3 to obtain a similar conclusion for maps in a neighborhood of $f$ in $\mathscr{A}$. Indeed, we will prove the following lemma.

Lemma 2.4. Let us consider a map $f \in \mathscr{A}$ such that $(\sqrt{|D f|})^{-1}$ is convex and let us consider two real numbers $c_{1}$ and $d_{1}, 0<c_{1}<d_{1}<1$. Then there is $C_{1}=C_{1}\left(f, c_{1}, d_{1}\right)>0$ such that for all $N \in \mathbb{N}$ there is a neighborhood $\mathscr{V}_{1}=$ $\mathscr{V}_{1}\left(f, N, c_{1}, d_{1}\right)$ of $f$ in $\mathscr{A}$ such that for all integers $l \leq N, g \in \mathscr{V}_{1}$ and $x \in \operatorname{dom}\left(g^{l}\right)$ such that $g^{l}(x) \in\left[c_{1}, d_{1}\right]$ we have that

$$
\left|D g^{l}(x)\right| \geq C_{1} \text {. }
$$

Proof. Fix a map $f \in \mathscr{A}$ such that $(\sqrt{|D f|})^{-1}$ is convex and $c_{1}$ and $d_{1}$ are as in the statement of the lemma.

Take the real numbers $c$ and $d$ with $0<c<c_{1}$ and $d_{1}<d<1$. It follows from the definition of the topology of $\mathscr{A}$ that for all $j \geq 1$ there is a neighborhood $\overline{\mathscr{V}}(j)$ of $f$ in $\mathscr{A}$ such that if $g \in \overline{\mathscr{V}}(j), w \in \operatorname{dom}\left(g^{j}\right)$ and $g^{j}(w) \in\left[c_{1}, d_{1}\right]$, then $w \in \operatorname{dom}\left(f^{j}\right)$ and

$$
f^{j}(w) \in[c, d] .
$$

To see this, we extend the functions of $\mathscr{A}$ to endomorphisms at some interval as is shown in Figure 3 .

Take $C_{0}$ given by Lemma 2.3 applied to $f, c$ and $d$ as above. It follows from the definition of the topology of $\mathscr{A}$ that for all $i \geq 1$ there is a neighborhood $\tilde{\mathscr{V}}(i)$ of $f$ in $\mathscr{A}$ such that if $g \in \tilde{\mathscr{V}}(i), z \in[0,1]$, then

$$
\left|D f^{i}(z)-D g^{i}(z)\right|<\frac{C_{0}}{2} .
$$




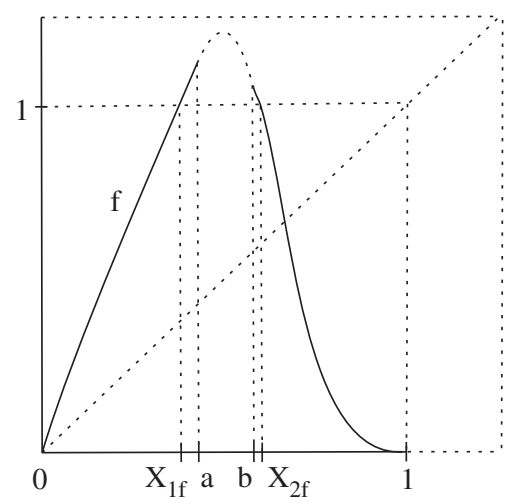

Figure 3. Extension of $f \in \mathscr{A}$ to an endomorphism at the interval.

Now fix an integer $N \geq 1$. Define

$$
\overline{\mathscr{U}}=\overline{\mathscr{U}}(f, N)=\bigcap_{1 \leq j \leq N} \overline{\mathscr{V}}(j)
$$

and

$$
\tilde{\mathscr{U}}=\tilde{\mathscr{U}}(f, N)=\bigcap_{1 \leq i \leq N} \tilde{\mathscr{V}}(i) .
$$

Define

$$
\mathscr{V}_{1}=\overline{\mathscr{U}} \cap \tilde{\mathscr{U}} \text {. }
$$

Taking $C_{1}=\frac{C_{0}}{2}$, let us prove that the neighborhood $\mathscr{V}_{1}$ works. For this we fix an integer $1 \leq l \leq N, g \in \mathscr{V}_{1}$ and $x \in \operatorname{dom}\left(g^{l}\right)$ such that $g^{l}(x) \in\left[c_{1}, d_{1}\right]$. In particular, if $g \in \overline{\mathscr{U}}$, then (2.5) implies that $f^{l}(x) \in[c, d]$ (taking $j=l$ ). Moreover, as in particular $g \in \tilde{\mathscr{U}}$, then (2.6) implies $\left|D f^{l}(x)-D g^{l}(x)\right|<\frac{C_{0}}{2}$ (taking $i=l$ ). As $f^{l}(x) \in[c, d]$, (2.1) in Lemma 2.3 (taking $i=l$ ) implies that

$$
\left|D f^{l}(x)\right| \geq C_{0} .
$$

Therefore, (2.7) implies that

$$
\begin{aligned}
\left|D g^{l}(x)\right| & =\left|D g^{l}(x)-D f^{l}(x)+D f^{l}(x)\right| \\
& \geq\left|D f^{l}(x)\right|-\left|D g^{l}(x)-D f^{l}(x)\right| \\
& \geq C_{0}-\frac{C_{0}}{2} \\
& =\frac{C_{0}}{2}=C_{1},
\end{aligned}
$$

which completes the proof.

Definition 2.5. Let us consider $f \in \mathscr{A}$. An $f$-invariant subset $K \subset \operatorname{dom}(f)$ is said to be a hyperbolic set for $f$ if there are constants $C>0$ and $\lambda>1$ such that for every $x \in K$ and every $n \in \mathbb{N}$,

$$
\left|D f^{k}(x)\right|>C \lambda^{n} \text {. }
$$

We say that $f$ is hyperbolic in $K$ if $K$ is a hyperbolic set for $f$. 
The following lemma is an easy characterization for compact invariant hyperbolic sets (e.g., Lemma 2.1, p. 220, [19]).

Proposition 2.6. Let us consider $f \in \mathscr{A}$ and $K$ an $f$-invariant compact set. Then, $K$ is hyperbolic for $f$ if only if for each $x \in K$ there exists an integer $n=n(x)$ such that $\left|D f^{n}(x)\right|>1$.

We say that $c$ is a critical point of $f \in \mathscr{A}$ if $D f(c)=0$.

Definition 2.7. Let us consider $f \in \mathscr{A}$ and $c$ a critical point of $f$. We say that $f$ is hyperbolic far away from $c$ if for any $\delta>0, f$ is hyperbolic in the maximal $f$-invariant set contained in the complement of a neighborhood of size $\delta$ around $c$.

First of all we note that a map $f \in \mathscr{A}$ with $(\sqrt{|D f|})^{-1}$ convex is hyperbolic far away from 1. In fact, fix a map $f \in \mathscr{A}$ with $(\sqrt{|D f|})^{-1}$ convex. For any $\delta>0$ let $(1-\delta, 1]$ be a neighborhood of size $\delta$ around 1 . We define

$$
\begin{aligned}
V(\delta) & =[0, a] \cup[b, 1-\delta], \\
W_{f}^{k}(\delta) & =\left\{x \in V(\delta) \mid f^{i}(x) \in V(\delta), i=0, \ldots, k-1\right\}, \quad \forall k \geq 1, \\
\Lambda_{f}(\delta) & =\bigcap_{k \geq 1} W_{f}^{k} .
\end{aligned}
$$

By definition, $\Lambda_{f}(\delta)$ is a forward-invariant compact set that does not contain the critical point 1. For maps in $\mathscr{A}$ such that for all $i,\left|D f^{i}\right|$ satisfies the Weak Minimum Principle in Lemma 2.2, Singer's Theorem (see [19], p. 155; see also [28]) and Misiurewicz's Theorem (see [19, p. 231) are still valid, so we have that $\Lambda_{f}(\delta)$ is a hyperbolic set. To show that this property remains valid in a neighborhood of $f$, we follow ideas from Mañe's Hyperbolicity Theorem.

Lemma 2.8. For every map $f \in \mathscr{A}$ such that $(\sqrt{|D f|})^{-1}$ is convex, there are $\delta_{2}=\delta_{2}(f)>0$ and a constant $C_{2}=C_{2}(f)>0$ satisfies the following property: for all $0<\delta<\delta_{2}$ there are $\lambda_{2}=\lambda_{2}(f, \delta)>1$ and a neighborhood $\mathscr{V}_{2}=\mathscr{V}_{2}(f, \delta)$ of $f$ in $\mathscr{A}$ such that if $g \in \mathscr{V}_{2}$ and for $k \in \mathbb{N}, x \in \operatorname{dom}\left(g^{k}\right)$ satisfies that $x, g(x), \ldots, g^{k-1}(x) \notin$ $(1-\delta, 1]$ and $g^{k}(x) \in\left[1-\delta_{2}, 1\right]$, then

$$
\left|D g^{k}(x)\right| \geq C_{2} \lambda_{2}^{k} \text {. }
$$

Proof. Let us consider a map $f \in \mathscr{A}$ with $(\sqrt{|D f|})^{-1}$ convex. Choose $\delta_{2}>0, c_{1}, d_{1}$ with $0<c_{1}<d_{1}<1$ and a neighborhood $\overline{\mathscr{V}}_{2}$ of $f$ in $\mathscr{A}$ in such way that if $g \in \overline{\mathscr{V}}_{2}$ and $x \in \operatorname{dom}(g)$ satisfy $g(x) \geq 1-\delta_{2}$, then $x \in\left[c_{1}, d_{1}\right]$.

Let $C_{1}$ be as in Lemma 2.4 applied to $f, c_{1}$ and $d_{1}$ chosen as above. Let us consider $\tilde{C}_{1}<\min \left\{|D f(x)|: x \in\left[c_{1}, d_{1}\right]\right\}$. Shrinking $\overline{\mathscr{V}}_{2}$, we can suppose that for all $g \in \overline{\mathscr{V}}_{2}$, for all $x \in\left[c_{1}, d_{1}\right]$ then $|D g(x)|>\tilde{C}_{1}$. Define

$$
C_{2}=\min \left\{1, \frac{C_{1} \cdot \tilde{C}_{1}}{2}\right\} .
$$

Now fix $\delta, 0<\delta<\delta_{2}$. For such a $\delta$ we shall find $\mathscr{V}_{2}$ and $\lambda_{2}$ as follows:

For all $h \in \mathscr{A}$, we define the auxiliary sets

$$
\begin{aligned}
V(\delta) & =[0, a] \cup[b, 1-\delta], \\
W_{h}^{k}(\delta) & =\left\{x \in V(\delta) \mid h^{i}(x) \in V(\delta), i=0, \ldots, k-1\right\}, \quad \forall k \geq 1, \\
\Lambda_{h}(\delta) & =\bigcap_{k \geq 1} W_{h}^{k} .
\end{aligned}
$$


As we just observed, by Singer's and Misiurewicz's theorems we have that $\Lambda_{f}(\delta)$ is a hyperbolic set. From this it follows that there are positive constants $\hat{C}=$ $\hat{C}(f, \delta)>0$ and $\hat{\lambda}=\hat{\lambda}(f, \delta)>1$ such that for all $k \in \mathbb{N}$ and $x \in \Lambda_{f}(\delta)$ one has

$$
\left|D f^{k}(x)\right| \geq \hat{C} \hat{\lambda}^{k} \text {. }
$$

Then, from the openness of the hyperbolicity we can find a neighborhood $\tilde{\mathscr{V}}=$ $\tilde{\mathscr{V}}(f, \delta)$ of $f$ in $\mathscr{A}$ and constants $\tilde{C}=\tilde{C}(f, \delta), \tilde{\lambda}=\tilde{\lambda}(f, \delta)$, with $\tilde{C}>0$ and $\hat{\lambda}>\tilde{\lambda}>1$ such that if $g \in \tilde{\mathscr{V}}$ and $x$ satisfy $g^{i}(x) \in V(\delta)$ for all $0 \leq i \leq k-1$, then

$$
\left|D g^{k}(x)\right| \geq \tilde{C} \tilde{\lambda}^{k}
$$

From (2.9) we can find $K=K(f, \delta) \in \mathbb{N}$ and $\hat{\lambda}>\hat{\lambda}_{2}=\hat{\lambda}_{2}(f, \delta)>1$ such that if $k \geq K, g \in \tilde{\mathscr{V}}$ and $x$ satisfy $g^{i}(x) \in V(\delta)$ for all $0 \leq i \leq k-1$, then

$$
\left|D g^{k}(x)\right| \geq \hat{\lambda}_{2}^{k}
$$

(Just take $K=\min \left\{k: \tilde{C} \tilde{\lambda}^{k}>1\right\}$ and $\hat{\lambda}_{2}$ such that $1<\hat{\lambda}_{2}<\min \left\{\tilde{\lambda} \tilde{C}^{\frac{1}{K}}, \tilde{\lambda}\right\}$.)

Let $\mathscr{V}_{1}$ be the neighborhood of $f$ in $\mathscr{A}$ given by Lemma 2.4 for this $K$.

Let us consider $\lambda_{2}=\lambda_{2}(f, \delta), 1<\lambda_{2}<\hat{\lambda}_{2}$ such that

$$
\lambda_{2}^{K}<2
$$

We show that the lemma works with $\mathscr{V}_{2}=\mathscr{V}_{2}(f, \delta)=\overline{\mathscr{V}}_{2} \cap \tilde{\mathscr{V}} \cap \mathscr{V}_{1}$ and $\lambda_{2}$ as was chosen.

Fix $g \in \mathscr{V}_{2}, k \in \mathbb{N}$ and $x \in \operatorname{dom}\left(g^{k}\right)$ satisfying $x, g(x), \ldots, g^{k-1}(x) \notin(1-\delta, 1]$ and $g^{k}(x) \in\left[1-\delta_{2}, 1\right]$.

If $k \geq K$, then (2.10) and the definitions of $\lambda_{2}$ and $C_{2}$ imply that

$$
\left|D g^{k}(x)\right| \geq \hat{\lambda}_{2}^{k} \geq C_{2} \lambda_{2}^{k}
$$

If $k<K$, then (2.4) of Lemma 2.4 because $g^{k-1}(x)$ belongs to $\left[c_{1}, d_{1}\right]$, (2.11) and the definitions of $\lambda_{2}$ and $C_{2}$ imply that

$$
\begin{aligned}
\left|D g^{k}(x)\right| & =\left|D g^{k-1}(x)\right| \cdot\left|D g\left(g^{k-1}(x)\right)\right| \\
& \geq C_{1} \cdot \tilde{C}_{1} \\
& =\frac{C_{1} \cdot \tilde{C}_{1}}{2} 2 \\
& >\frac{C_{1} \cdot \tilde{C}_{1}}{2} \lambda_{2}^{K} \\
& \geq C_{2} \lambda_{2}^{k}
\end{aligned}
$$

Finally, (2.12) and (2.13) imply (2.8) of Lemma 2.8. Therefore, the lemma follows.

The following lemma shows that points close to the critical point retrieve the derivative in a very fast way. The arguments to prove it resemble the ones used in Lemma 1.1, p. 249 in 25.

Lemma 2.9. For every $f \in \mathscr{A}$ and $0<C \leq 1$ there are a neighborhood $\mathscr{V}_{3}=$ $\mathscr{V}_{3}(f, C)$ of $f$ in $\mathscr{A}$, constants $\delta_{3}=\delta_{3}(f, C)>0, \lambda_{3}=\lambda_{3}(f, C)>1$ and $L \in \mathbb{N}$ with $C \lambda_{3}^{L}>1$ such that for all $g \in \mathscr{V}_{3}$, for all $x \in\left(1-\delta_{3}, 1\right)$, there is an integer $l_{g}(x)>L$ such that $g^{j}(x) \notin\left(1-\delta_{3}, 1\right], j=1, \ldots, l_{g}(x)-1$ and

$$
\left|D g^{l_{g}(x)}(x)\right| \geq \lambda_{3}^{l_{g}(x)} .
$$


Proof. Fix $f \in \mathscr{A}$ and $0<C \leq 1$. For every $\eta>0$ we consider the neighborhood for $f$ of size $\eta$ in $\mathscr{A}$, that is,

$$
\mathscr{V}_{\eta}=\left\{g \in \mathscr{A}: d_{\mathscr{A}}(f, g)<\eta\right\} .
$$

Then

$$
\forall z \in[0, a] \cup[b, 1], \quad \forall g \in \mathscr{V}_{\eta}, \text { we have }|D f(z)|-\eta \leq|D g(z)| \leq|D f(z)|+\eta .
$$

Moreover, we take $\varepsilon$ small enough such that

$$
|z| \leq \varepsilon \Longrightarrow|D f(0)|-\eta \leq|D f(z)| \leq|D f(0)|+\eta .
$$

Then $\forall g \in \mathscr{V}_{\eta}$ and $|z| \leq \varepsilon$ we have that

$$
|D f(0)|-2 \eta \leq|D g(z)| \leq|D f(0)|+2 \eta .
$$

Put

$$
\begin{aligned}
& m_{\eta}=|D f(0)|-2 \eta, \\
& M_{\eta}=|D f(0)|+2 \eta .
\end{aligned}
$$

Because $D f(0)>1$ we can choose $m, M$ with $1<m<D f(0)<M$ such that

$$
m M^{\frac{1-\alpha_{f}}{\alpha_{f}}}>1 \text {. }
$$

From this, shrinking $\eta$ if necessary, because $\alpha_{g}$ is close to $\alpha_{f}$, there is $\hat{\lambda}=\hat{\lambda}(f)>$ 1 depending only on $f$ such that for all $g \in \mathscr{V}_{\eta}$,

$$
m M^{\frac{1-\alpha_{g}}{\alpha_{g}}}>\hat{\lambda}>1 \text {. }
$$

Moreover if $\eta$ is small enough we have that

$$
m<m_{\eta}<M_{\eta}<M \text {. }
$$

As $f(1)=0$ we can choose $0<\hat{\delta}=\hat{\delta}(f)<1$ such that $0<f(x)<\frac{\varepsilon}{2}$ for all $x \in(1-\hat{\delta}, 1)$. Shrinking $\eta$ again, we can assume that $0<g(x)<\varepsilon$ for all $x \in(1-\hat{\delta}, 1)$ and for all $g \in \mathscr{V}_{\eta}$.

For $g \in \mathscr{V}_{\eta}$ and $x \in[1-\hat{\delta}, 1)$ we define

$$
l=l_{g}(x)=\min \left\{j \geq 1: g^{j}(g(x)) \geq \varepsilon\right\} .
$$

Note that for all $x \in(1-\hat{\delta}, 1), l_{g}(x) \geq l_{g}(1-\hat{\delta})$ because of the monotonicity of $g$ in the intervals $[0, a]$ and $[b, 1]$.

To choose $\lambda_{3}$ we need to make some estimates. Let us consider $g \in \mathscr{V}_{\eta}$ and $x \in(1-\hat{\delta}, 1)$. Denote $z=g(x)$. By definition of $l=l_{g}(x)$ in (2.17) we have that $z, g(z), \ldots, g^{l-1}(z) \in[0, \varepsilon)$ and

$$
g^{l}(z) \geq \varepsilon
$$

As $g^{l}(0)=0$, then (2.16) and the Mean Value Theorem imply that there is $\xi \in(0, z)$ such that

$$
g^{l}(z)=D g^{l}(\xi) z \leq M^{l} z .
$$

Replacing this inequality in (2.18) we get

$$
M^{l} z \geq \varepsilon,
$$

that is,

$$
z \geq \varepsilon M^{-l} .
$$


By properties of $f$ we have that there exists a positive constant $\hat{K}_{f}$ such that $\frac{1}{\hat{K}_{f}} \leq\left|H_{f}(x)\right| \leq \hat{K}_{f}$. Using that $\left|H_{f}(x)-H_{g}(x)\right|<\eta$ we obtain that $\left|H_{g}(x)\right|<$ $\hat{K}_{f}+\eta$. Moreover, as $g(x)=H_{g}(x)|x-1|^{\alpha_{g}}=z$ we have that

$$
z \leq\left(\hat{K}_{f}+\eta\right)|x-1|^{\alpha_{g}}
$$

This implies that

$$
|x-1|^{\alpha_{g}-1} \geq\left(\frac{z}{\hat{K}_{f}+\eta}\right)^{\frac{\alpha_{g}-1}{\alpha_{g}}} .
$$

Then (2.19) and (2.20) imply that

$$
\begin{aligned}
|x-1|^{\alpha_{g}-1} & \geq\left(\frac{z}{\hat{K}_{f}+\eta}\right)^{\frac{\alpha_{g}-1}{\alpha_{g}}} \\
& =z^{\frac{\alpha_{g}-1}{\alpha_{g}}} \frac{1}{\left(\hat{K}_{f}+\eta\right)^{\frac{\alpha_{g}-1}{\alpha_{g}}}} \\
& \geq\left(\frac{\varepsilon}{\hat{K}_{f}+\eta}\right)^{\frac{\alpha_{g}-1}{\alpha_{g}}}\left(M^{\frac{1-\alpha_{g}}{\alpha_{g}}}\right)^{l} .
\end{aligned}
$$

As $H_{g}(1) \neq 0$ and $D g(x)=|x-1|^{\alpha_{g}-1}\left(-\alpha_{g} H_{g}(x)+|x-1| D H_{g}(x)\right)$, shrinking $\hat{\delta}$, we can choose a positive $\tilde{K}_{f}$ such that

$$
|D g(x)| \geq \tilde{K}_{f}|x-1|^{\alpha_{g}-1} .
$$

Indeed, by hypothesis we have that $\left|\alpha_{f}-\alpha_{g}\right|<\eta$. $\left|H_{f}(x)-H_{g}(x)\right|<\eta$ and

$$
\left|(x-1)\left(D H_{f}(x)-D H_{g}(x)\right)\right|<\eta \text {. }
$$

Moreover, we can take $\hat{\delta}$ such that $\left|(x-1) D H_{f}(x)\right|<\varepsilon$ for all $x \in[1-\hat{\delta}, 1)$. So we get

$$
\begin{aligned}
|D g(x)| & =|x-1|^{\alpha_{g}-1}\left|-\alpha_{g} H_{g}(x)+\right| x-1\left|D H_{g}(x)\right| \\
& \geq|x-1|^{\alpha_{g}-1}\left(\alpha_{g}\left|H_{g}(x)\right|-|x-1|\left|D H_{g}(x)\right|\right) \\
& >|x-1|^{\alpha_{g}-1}\left(\left(\frac{1}{\hat{K}_{f}}-\eta\right)\left(\alpha_{f}-\eta\right)-\eta-\varepsilon\right) \\
& =\tilde{K}_{f}|x-1|^{\alpha_{g}-1}
\end{aligned}
$$

where $0<\tilde{K}_{f}<\lim _{(\eta, \varepsilon) \rightarrow(0,0)}\left(\frac{1}{\hat{K}_{f}}-\eta\right)\left(\alpha_{f}-\eta\right)-\eta-\varepsilon=\frac{\alpha_{f}}{\hat{K}_{f}}$.

From the Chain Rule, (2.22) and (2.16) we have

$$
\left|D g^{l}(x)\right|=\left|D g^{l-1}(g(x))\right||D g(x)| \geq \tilde{K}_{f}|x-1|^{\alpha_{g}-1} m^{l-1} .
$$


Moreover, the Chain Rule, (2.23) and (2.21) yield

$$
\begin{aligned}
\left|D g^{l}(x)\right| & \geq \tilde{K}_{f}|x-1|^{\alpha_{g}-1} m^{l-1} \\
& \geq \tilde{K}_{f}\left(\frac{\varepsilon}{\hat{K}_{f}+\eta}\right)^{\frac{\alpha_{g}-1}{\alpha_{g}}}\left(M^{\frac{1-\alpha_{g}}{\alpha_{g}}}\right)^{l} m^{l-1} \\
& =\frac{\tilde{K}_{f}}{m}\left(\frac{\varepsilon}{\hat{K}_{f}+\eta}\right)^{\frac{\alpha_{g}-1}{\alpha_{g}}}\left(m M^{\frac{1-\alpha_{g}}{\alpha_{g}}}\right)^{l} .
\end{aligned}
$$

Using (2.15) and (2.24) we get

$$
\begin{aligned}
\left|D g^{l}(x)\right| & \geq \frac{\tilde{K}_{f}}{m}\left(\frac{\varepsilon}{\hat{K}_{f}+\eta}\right)^{\frac{\alpha_{f}-1+\eta}{\alpha_{f}-\eta}} \hat{\lambda}^{l} \\
& =C_{\varepsilon, f} \cdot \hat{\lambda}^{l}
\end{aligned}
$$

where

$$
C_{\varepsilon, f}=\frac{\tilde{K}_{f}}{m}\left(\frac{\varepsilon}{\hat{K}_{f}+\eta}\right)^{\frac{\alpha_{f}-1+\eta}{\alpha_{f}-\eta}} .
$$

We claim that there exists $L_{0} \in \mathbb{N}$ with $C_{\varepsilon, f} \hat{\lambda}^{L_{0}}>1$ such that $l>L_{0}$. Indeed, using (2.19) we obtain that for all $x \in[1-\hat{\delta}, 1)$,

$$
l_{g}(x) \geq l_{g}(1-\hat{\delta})>\frac{\ln (\varepsilon)-\ln (g(1-\hat{\delta}))}{\ln (M)} .
$$

Take $L_{0} \in \mathbb{N}$ such that $C_{\varepsilon, f} \hat{\lambda}^{L_{0}}>1$. As $f(1)=0$, we can choose $\eta$ and $\hat{\delta}$ in such a way that for all $g \in \mathscr{V}_{\eta}$,

$$
\frac{\ln (\varepsilon)-\ln (g(1-\hat{\delta}))}{\ln (M)}>L_{0} .
$$

Then, from (2.26) for all $x \in[1-\hat{\delta}, 1), l=l_{g}(x)>L_{0}$, proving the claim.

Take $\lambda_{3}, 1<\lambda_{3}<\min \left\{\left(C_{\varepsilon, f}\right)^{\frac{1}{L_{0}}} \hat{\lambda}, \hat{\lambda}\right\}$. Following the same arguments as above we obtain an integer $L$ with $C \lambda_{3}^{L}>1$ such that $l>L$.

Therefore, (2.25) implies that

$$
\begin{aligned}
\left|D g^{l}(x)\right| & \geq C_{\varepsilon, f} \hat{\lambda}^{l} \\
& =C_{\varepsilon, f} \hat{\lambda}^{L_{0}} \hat{\lambda}^{l-L_{0}} \\
& \geq \lambda_{3}^{L_{0}} \lambda_{3}^{l-L_{0}} \\
& =\lambda_{3}{ }^{l},
\end{aligned}
$$

The lemma works with $\mathscr{V}_{\eta}$ for the chosen $\eta$ and $\delta_{3}=\hat{\delta}$. This ends the proof.

The following proposition shows that for a map $f \in \mathscr{A}$ with $(\sqrt{|D f|})^{-1}$ convex, times of return to a neighborhood of the critical point have hyperbolic behavior and that this remains valid for all maps $C^{1}$-close to $f$. The proposition recalls the Quasi-hyperbolicity Theorem for Misiurewicz maps and its extension to an open neighborhood (see [19, Theorem 6.3, p. 261 and Theorem 6.4, p. 262), but in our case the neighborhood of $f$ does not depend on the neighborhood of the critical point as in the before cited theorems. 
Proposition 2.10. Let us consider a map $f \in \mathscr{A}$ with $(\sqrt{|D f|})^{-1}$ convex. Then there are a neighborhood $\mathscr{V}_{4}=\mathscr{V}_{4}(f)$ of $f$ in $\mathscr{A}$ and constants $C_{4}=C_{4}(f)>0$, $\delta_{4}=\delta_{4}(f)>0, \lambda_{4}=\lambda_{4}(f)>1$ satisfying the following properties: If $k \in \mathbb{N}, g \in \mathscr{V}_{4}$ and $x \in \operatorname{dom}\left(g^{k}\right)$ are such that $g^{k}(x) \in\left(1-\delta_{4}, 1\right]$, then

$$
\left|D g^{k}(x)\right| \geq C_{4} \lambda_{4}^{k} \text {. }
$$

Proof. Fix a map $f \in \mathscr{A}$ with $(\sqrt{|D f|})^{-1}$ convex. Let us consider $C_{2}>0$ and $\delta_{2}$ given in Lemma 2.8 applied for $f$.

Take $C_{4}=\min \left\{1, C_{2}\right\}$. Applying Lemma 2.9 for $f$ and $C=C_{4}$ we obtain a neighborhood $\mathscr{V}_{3}$, the real numbers $\delta_{3}$ and $\lambda_{3}$ and an integer $L$. Choose $\delta_{4}$ such that $0<\delta_{4}<\min \left\{\delta_{2}, \delta_{3}\right\}$. By the conclusion of Lemma 2.8 applied to $\delta=\delta_{4}$, there are $\lambda_{2}$ and a neighborhood $\mathscr{V}_{2}$. Let us consider $\mathscr{V}_{4}=\mathscr{V}_{2} \cap \mathscr{V}_{3}$ and choose $\lambda_{4}$ in such a way that $1<\lambda_{4}<\min \left\{C_{2}^{\frac{1}{L}} \lambda_{3}, \lambda_{2}\right\}$. Note that $C_{2}^{\frac{1}{L}} \lambda_{3}>1$ because $C_{2}>C=C_{4}$.

Now we prove that the proposition works with $\mathscr{V}_{4}, C_{4}, \delta_{4}$ and $\lambda_{4}$ as chosen above.

Fix $g \in \mathscr{V}_{4}, k \in \mathbb{N}$ and $x \in \operatorname{dom}\left(g^{k}\right)$ and such that $g^{k}(x) \in\left(1-\delta_{4}, 1\right]$.

We decompose the orbit $\left\{g^{i}(x)\right\}_{i=0}^{k}$ into several blocks as follows:

$$
\begin{gathered}
\left\{x=x_{1}, g\left(x_{1}\right), \ldots, g^{k_{1}-1}\left(x_{1}\right)\right\},\left\{y_{1}=g^{k_{1}}\left(x_{1}\right), g\left(y_{1}\right), \ldots, g^{l_{1}-1}\left(y_{1}\right)\right\}, \\
\left\{x_{2}=g^{l_{1}}\left(y_{1}\right), g\left(x_{2}\right), \ldots, g^{k_{2}-1}\left(x_{2}\right)\right\},\left\{y_{2}=g^{k_{2}}\left(x_{2}\right), g\left(y_{2}\right), \ldots, g^{l_{2}-1}\left(y_{2}\right)=x_{3}\right\}, \ldots, \\
\left\{x_{m}=g^{l_{m}-1}\left(y_{m-1}\right), g\left(x_{m}\right), \ldots, g^{k_{m}}\left(x_{m}\right)=y_{m}=g^{k}(x)\right\}
\end{gathered}
$$

where $k_{1}$ is the first integer such that $g^{k_{1}}\left(x_{1}\right) \in\left(1-\delta_{4}, 1\right), l_{1} \geq L$ is given by the conclusion of Lemma 2.9 applied to $y_{1}, k_{2}$ is the first integer such that $g^{k_{2}}\left(x_{2}\right) \in$ $\left(1-\delta_{4}, 1\right)$, and so on.

Notice that $k_{1}+l_{1}+\cdots+k_{m-1}+l_{m-1}+k_{m}=k$.

Using the Chain Rule Theorem, (2.8) of Lemma 2.8, (2.14) of Lemma 2.9, and the definitions of $C_{4}$ and $\lambda_{4}$ we obtain

$$
\begin{aligned}
\left|D g^{k}(x)\right| & =\left|D g^{k_{m}}\left(x_{m}\right)\right| \ldots\left|D g^{k_{2}}\left(y_{2}\right)\right|\left|D g^{k_{2}}\left(x_{2}\right)\right|\left|D g^{l_{1}}\left(y_{1}\right)\right|\left|D g^{k_{1}}\left(x_{1}\right)\right| \\
& \geq\left(C_{2} \lambda_{2}^{k_{m}}\right) \ldots \lambda_{3}^{l_{2}}\left(C_{2} \lambda_{2}^{k_{2}}\right) \lambda_{3}^{l_{1}}\left(C_{2} \lambda_{2}^{k_{1}}\right) \\
& =C_{2} \lambda_{2}^{k_{1}+\cdots+k_{m}}\left(C_{2} \lambda_{3}^{l_{1}}\right) \ldots\left(C_{2} \lambda_{3}^{l_{m-1}}\right) \\
& \geq C_{2} \lambda_{2}^{k_{1}+\cdots+k_{m}} \hat{\lambda}^{l_{1}} \ldots \hat{\lambda}^{l_{m-1}} \\
& \geq C_{2} \lambda_{4}^{k} \\
& \geq C_{4} \lambda_{4}^{k}
\end{aligned}
$$

This proves (2.28) of Proposition 2.10.

Remark 2.11. Notice that for $g \in \mathscr{V}_{4}, k \geq 1$ and $x \in\left(1-\delta_{4}, 1\right)$ such that $g^{k}(x) \in$ $\left(1-\delta_{4}, 1\right]$ the proof of Proposition 2.10 gives us:

$$
\left|D g^{k}(x)\right| \geq \lambda_{4}^{k}
$$

Corollary 2.12. Let us consider a map $f \in \mathscr{A}$ with $(\sqrt{|D f|})^{-1}$ convex. Then there exists a neighborhood $\mathscr{V}_{5}=\mathscr{V}_{5}(f)$ of $f$ in $\mathscr{A}$ such that each $g \in \mathscr{V}_{5}$ is hyperbolic far away from the critical point 1.

Proof. Let us consider a map $f \in \mathscr{A}$ with $(\sqrt{|D f|})^{-1}$ convex. Let $\delta_{4}$ and neighborhood $\mathscr{V}_{4}$ be as given by Proposition 2.10. As we just observed before we have that $f$ is hyperbolic far away from 1 , so $f$ is hyperbolic in the maximal $f$-invariant set in the complement of $\left(1-\delta_{4}, 1\right]$. By shrinking $\mathscr{V}_{4}$ if necessary we can assume 
that every $g \in \mathscr{V}_{4}$ is hyperbolic in the maximal $g$-invariant set in the complement of $\left(1-\delta_{4}, 1\right]$ (indeed, in the proof of Proposition 2.10, it can be seen that $\mathscr{V}_{4}$ just satisfies this property). Define $\mathscr{V}_{5}=\mathscr{V}_{4}$. Now take $g \in \mathscr{V}_{5}$ and $\delta<\delta_{4}$. Then, for all $x$ in the maximal $g$-invariant contained in $[0,1-\delta]$ we have that either $\forall k_{0}>1, g^{k_{0}}(x) \notin\left(1-\delta_{4}, 1\right]$ or for some $k_{1}>1, g^{k_{1}}(x) \in\left(1-\delta_{4}, 1\right]$. In the first case, taking $k_{0}$ big enough we have that $\left|D g^{k_{0}}(x)\right|>1$. In the other case, $\left|D g^{k_{1}}(x)\right|>1$ (see Remark 2.11). From this and Proposition 2.6 we obtain that $g$ is hyperbolic in the maximal $g$-invariant set in the complement of $(1-\delta, 1]$. As $\delta$ is arbitrary, then $g$ is hyperbolic far away from the critical point 1.

The following theorem is related with nonexistence of wandering intervals. This old problem goes back to Poincaré's work dealing with homeomorphisms of the circle (see 22]). Since then efforts of a number of authors have been directed towards proving the nonexistence of wandering intervals because their appearance complicates the understanding of the dynamics.

Theorem 2.13. Let us consider a map $f \in \mathscr{A}$ with $(\sqrt{|D f|})^{-1}$ convex. Then there exists a neighborhood $\mathscr{V}=\mathscr{V}(f)$ of $f$ in $\mathscr{A}$ such that every $g \in \mathscr{V}$ is hyperbolic far away from the critical point 1 and the maximal $g$-invariant set contained in $I=[0,1] \Lambda_{g}=\bigcap_{i=0}^{\infty} g^{-i}(I)$ has no interval.

Proof. Fix a map $f \in \mathscr{A}$ with $(\sqrt{|D f|})^{-1}$ convex. Let us consider $\mathscr{V}_{4}, \delta_{4}$ and $\lambda_{4}$ given by Proposition 2.10. Take a neighborhood $\mathscr{V}=\mathscr{V}_{5} \subset \mathscr{V}_{4}$ of $f$ in $\mathscr{A}$ given by Corollary 2.12, Now fix $g \in \mathscr{V}$. First we observe that $g$ has no sinks. Indeed, by Corollary [2.12, $g$ is hyperbolic far away from the critical point 1.

Suppose that $\Lambda_{g}=\bigcap_{i=0}^{\infty} g^{-i}(I)$ contains an interval $J$. If there are integers $m \neq n$ such that $g^{m}(J) \cap g^{n}(J)$ has nonempty interior, then $g$ has sinks (see [8], Lemma A, p. 142) and so we get a contradiction. Therefore, the sequence of intervals $\left\{g^{n}(J)\right\}_{n=0}^{\infty}$ are pairwise disjoint and cannot accumulate in a sink; i.e. $J$ is a wandering interval. From this it follows that $g^{n}(J)$ accumulate to 1 and

$$
\left|g^{n}(J)\right| \rightarrow 0 \text { as } n \rightarrow+\infty .
$$

Let us consider $0<\eta<\delta_{4}$ and an integer $n_{0}$ in such a way that $\forall n \geq n_{0}$, $\left|g^{n}(J)\right|<\delta_{4}-\eta$. So, if for $n \geq n_{0}, g^{n}(J) \cap(1-\eta, 1) \neq \emptyset$, then $g^{n}(J) \subset\left(1-\delta_{4}, 1\right)$.

As the iterates of $J$ accumulate to 1 , there is a sequence $n_{k}$ such that $g^{n_{k}}(J) \subset$ $\left(1-\delta_{4}, 1\right)$. From the Chain Rule Theorem we have

$$
\begin{aligned}
\left|g^{n_{k}}(J)\right| & =\left|g^{n_{k}-n_{0}}\left(g^{n_{0}}(J)\right)\right| \\
& =\left|D g^{n_{k}-n_{0}}(\xi)\right|\left|g^{n_{0}}(J)\right|,
\end{aligned}
$$

for some $\xi \in g^{n_{0}}(J) \subset\left(1-\delta_{4}, 1\right)$.

Because $g^{n_{k}-n_{0}}(\xi) \in g^{n_{k}}(J) \subset\left[1-\delta_{4}, 1\right]$ we can apply (2.29) to obtain

$$
\left|g^{n_{k}}(J)\right| \geq \lambda_{2}^{n_{k}-n_{0}}\left|g^{n_{0}}(J)\right| \text {. }
$$

As $n_{k} \rightarrow \infty$ we have that

$$
\left|g^{n_{k}}(J)\right| \rightarrow \infty
$$

and so we get a contradiction with (2.30).

Proof of the Main Theorem. Fix a map $f \in \mathscr{A}$ with $(\sqrt{|D f|})^{-1}$ convex. Let us consider $\mathscr{U}=\mathscr{V}$, where $\mathscr{V}$ is the neighborhood of $f$ in $\mathscr{A}$ given by Theorem 2.13. 
From now on, we follow the classical arguments to prove that two maps on the interval having Markov partitions are conjugates (see by example [14, Lemma 2, p. 344]).

\section{ACKNOWLEDGMENT}

The authors want to thank the Instituto Nacional de Matemática Pura e Aplicada, IMPA-Brasil, for its hospitality.

\section{REFERENCES}

1. Blokh, A.M. and Lyubich, M.Yu., Nonexistence of wandering intervals and structure of topological attractors of one-dimensional dynamical systems. II. The smooth case. Ergodic Theory Dynam. Systems, 9 (1989), 751-758. MR.1036906 (91e:58101)

2. Blokh, A.M. and Lyubich, M.Yu., The absence of wandering intervals in one-dimensional smooth dynamical systems. Soviet Math. Dokl. 39 (1989), 169-172. MR.997178 (90f:58110)

3. Berry, D. and Mestel, B.D., Wandering intervals for Lorenz maps with bounded nonlinearity. Bull. London Math. Soc. 23 (1991), 183-189. MR.1122907 (93c:58119)

4. Cedervall, S., Invariant Measures and Correlation Decay for S-multimodal Interval Maps. Doctor of Philosophy Thesis, Imperial College of Science, Technology and Medicine, University of London, 2006.

5. Cobo, Milton, Piece-wise affine maps conjugate to interval exchanges. Ergodic Theory Dynam. Systems 22 (2002), no. 2, 375-407. MR.1898797 (2003h:37003)

6. Coven, E. M. and Nitecki, Z., Nonwandering sets of the powers of maps of the interval. Ergodic Theory Dynam. Systems 1 (1981), no. 1, 9-31. MR627784 (82m:58043)

7. Denjoy, A., Sur les courbes définies par les équations différentielles à la surface du tore. Journal de Mathématiques Pures et Appliquées 11 (1932), 333-375.

8. Guckenheimer, J., Sensitive dependence to initial conditions for one-dimensional maps. Commun. Math. Phys. 70 (1979), 133-160. MR553966 (82c:58037)

9. Guckenheimer, J. and Williams, R., The structure of Lorenz attractors. Publ. Math. IHES, 50 (1979), 73-99. MR.556583 (82b:58055b)

10. Hall, C.R., $A C^{\infty}$ Denjoy counterexample. Ergod. Th. and Dynam. Sys. 7 (1981), 509-530.

11. Harrison, J., Wandering intervals. Dynamical systems and turbulence. Lecture Notes in Math., 898, Springer, Berlin-New York, 1981, 154-163. MR654888 (83h:58059)

12. Ivanov, A.F., An example of infinitely many sinks for smooth interval maps. Acta Math. Univ. Comenian. (N.S.) 61 (1992), no. 1, 3-9. MR.1205854 (93m:58060)

13. Lyubich, M.Yu., Nonexistence of wandering intervals and structure of topological attractors of one-dimensional dynamical systems. I. The case of negative Schwarzian derivative. Ergodic Theory Dynam. Systems 9 (1989), 737-749. MR:1036905 (91e:58100)

14. Labarca, L. and Pacifico, M.J., Stability of singularity horseshoes. Topology 25 (1986), no. 3, 337-352. MR842429 (87h:58106)

15. de Melo, W., A finiteness problem for one-dimensional maps. Proc. Amer. Math. Soc. 101 (1987), 721-727. MR911040 (89a:58063)

16. Martens, M., de Melo, W., and van Strien, S., Julia-Fatou-Sullivan theory for real onedimensional dynamics. Acta Math. 168 (1992), no. 3-4, 273-318. MR1161268 (93d:58137)

17. de Melo, W. and van Strien, S., One-dimensional dynamics: the Schwarzian derivative and beyond. Bull. Amer. Math. Soc. (N.S.) 18 (1988), 159-162. MR.929092 (89e:58079)

18. de Melo, W. and van Strien, S., A structure theorem in one-dimensional dynamics. Ann. of Math. (2) 129 (1989), 519-546. MR997312 (90m:58106)

19. de Melo, W. and van Strien, S., One-dimensional dynamics. Springer-Verlag, Berlin, 1993. MR.1239171 (95a:58035)

20. Muñoz, E.M., San Martín, B. and Vera, J.A., Nonhyperbolic persistent attractors near the Morse-Smale boundary. Ann. Inst. Henri Poincaré Anal. Non Linéaire 20 (2003), 867-888. MR.1995505 (2004e:37033)

21. Milnor, J. and Thurston, W., On iterated maps of the interval. Lecture Notes in Math., 1342, Springer, Berlin, 1988, 465-563. MR 970571 (90a:58083)

22. Nitecki, Z., Differentiable dynamics. MIT Press, Cambridge, Mass.-London, 1971. MR0649788 (58:31210) 
23. Nowicki, T. and van Strien, S., Hyperbolicity properties of $C^{2}$ multi-modal Collet-Eckmann maps without Schwarzian derivative assumptions. Trans. Amer. Math. Soc. 321 (1990), no. 2, 793-810. MR994169 (91a:58097)

24. Parry, W., Symbolic dynamics and transformations of the unit interval. Trans. Amer. Math. Soc. 122 (1966), 368-378. MR0197683(33:5846)

25. Rovella, A., The dynamics of perturbations of the contracting Lorenz attractor. Bol. Soc. Brasil. Mat. (N.S.) 24 (1993), 233-259. MR1254985 (95a:58097)

26. Schwartz, A., A generalization of a Poincaré-Bendixson theorem to closed two-dimensional manifolds. Amer. J. Math. 85 (1963), 453-458. MR0155061 (27:5003)

27. Sharkovskiu, A.N. and Ivanov, A.F., $C^{\infty}$-mappings of an interval with attracting cycles with arbitrarily large periods. (Russian) Ukrain. Mat. Zh. 35 (1983), no. 4, 537-539. MR712483 (84m:58066)

28. Singer, D., Stable orbits and bifurcations of maps of the interval. SIAM J. Appl. Math. 35 (1978), 260-267. MR0494306 (58:13206)

29. van Strien, S. and Vargas, E., Real bounds, ergodicity and negative Schwarzian for multimodal maps. J. Amer. Math. Soc. 17 (2004), no. 4, 749-782. MR2083467|(2005i:37043)

30. Yoccoz, J.C., Il n'y a pas de contre-exemple de Denjoy analytique. C. R. Acad. Sci. Paris Sér. I Math. 298 (1984), no. 7, 141-144. MR741080 (85j:58134)

Departamento de Matemática, Facultad de Ciencias, Universidad del Bío Bío, Av. Collao \#1202, Casilla 5-C, Región de Concepción, Chile

E-mail address: dcarrasc@ubiobio.cl

Instituto de Matemáticas, Universidad Federal de Rio de Janeiro, P.O. Box 68530, 21945-970, RiO DE JANEIRO, BRASIL

E-mail address: morales@impa.br

Departamento de Matematicas, Universidad Católica del Norte, Av. Angamos 0610, Casilla 1280, Antofagasta, Chile

E-mail address: sanmarti@ucn.cl 